\title{
Correcting Frost Diagram Misconceptions Using Interactive Frost Diagrams
}

Kaitlyn G. Dutton and Mark C. Lipke*

Department of Chemistry and Chemical Biology, Rutgers, The State University of

5 New Jersey, Piscataway, New Jersey 08854, United States

\section{ABSTRACT}

Frost diagrams provide convenient illustrations of the aqueous reduction potentials and thermodynamic tendencies of different oxidation states of an element. Undergraduate textbooks often describe the lowest point on a Frost diagram as the most stable oxidation state of the element, but this interpretation is incorrect because the thermodynamic stability of each oxidation state depends on the specific redox conditions in solution (i.e., the potential applied by the environment or an electrode). Further confusion is caused by the widespread use of different, contradictory conventions for labeling the y-axis of these diagrams as either $n E^{\circ}$ or $-n E^{\circ}$, among other possibilities. To aid in discussing and correcting these common mistakes, we introduce a series of interactive Frost diagrams that illustrate the conditional dependence of the relative stabilities of each oxidation state of an element. We include instructor's notes for using these interactive diagrams and a written activity for students to complete using these diagrams.

GRAPHICAL ABSTRACT

Interactive Frost Diagrams

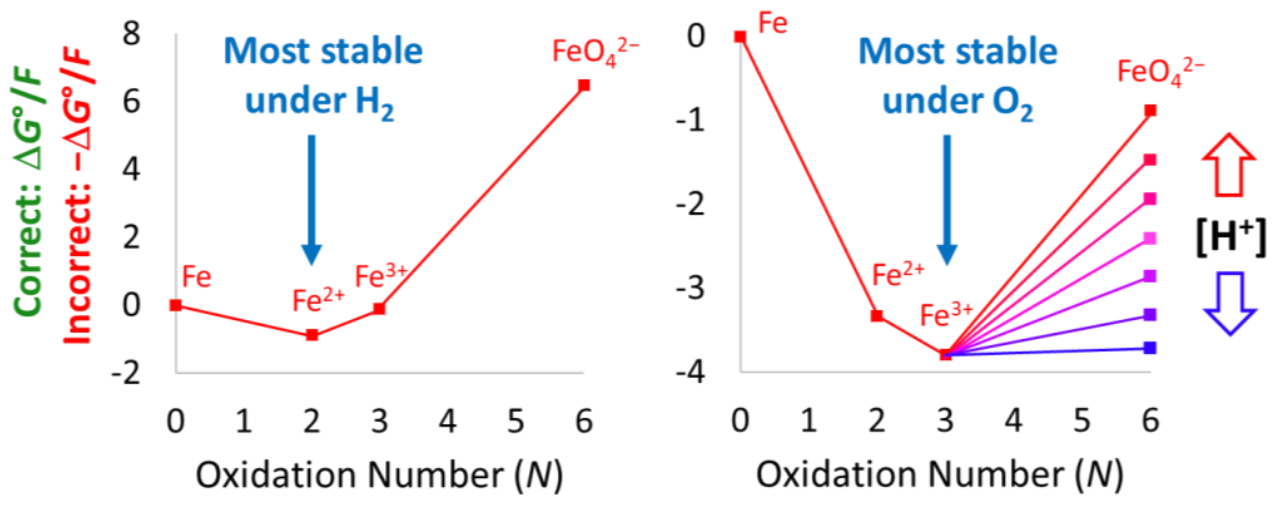

Variable Conditions - $p H$ and Applied Potentials

Undergraduate Instruction, Inorganic Chemistry, Redox, Frost Diagrams, Electrochemistry 
Frost diagrams ${ }^{1}$ provide a visual representation of reduction potentials and relative thermodynamic stabilities of different oxidation states of an element in aqueous conditions. In these

diagrams (Figure 1), the oxidation number $(N)$ of a common species (e.g., $\mathrm{Fe}^{2+}{ }_{a q}$ ) is plotted on the $\mathrm{x}$-axis and its free energy (or a proportional value) relative to the $N=0$ state is plotted on the y-axis. When the relative energy is provided as $\Delta G^{\circ} / \mathrm{F}$ (or $\Delta G / \mathrm{F}$ if $\mathrm{pH}$ $\neq 0$ or 14), the slopes connecting each point correspond to the reduction potentials $E^{\circ}$ (or $E$ for $\mathrm{pH} \neq 0$ or 14$)$ connecting these oxidation states owing to the relationship $\Delta G^{\circ}=-\mathrm{nF} E^{\circ}$. In this regard, Frost diagrams visually depict the electrode

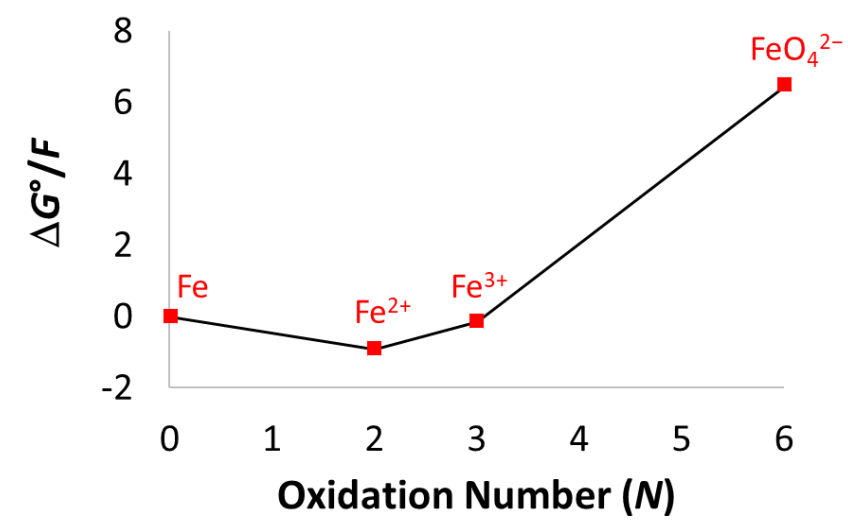

Figure 1. Frost diagrams for iron in $\mathrm{pH}=0$ solution. potentials tabulated in Latimer diagrams, ${ }^{2}$ and both are often taught alongside other common redox diagrams such as Pourbaix diagrams. ${ }^{3}$ Together, these graphs provide complementary depictions of the relationship between reduction potentials and the thermodynamics of redox processes. However, we have identified two notable points of confusion in common explanations of Frost diagrams.

Various textbooks and other resources utilize different, contradictory labels for the y-axis of Frost diagrams.4-18 As originally intended by Frost, ${ }^{1}$ the y-axis should be proportional to the free-energy change for conversion of the $N=0$ oxidation state of an element to a higher or lower oxidation state. However, in providing the free energy in terms of electrode potentials, authors have chosen to label the y-axis as either $n E^{\circ}$ or $-\mathrm{n} E^{\circ}$ to correspond to $\Delta G^{\circ} / \mathrm{F}$ ( $\mathrm{F}=$ Faraday's constant). Either option can be considered correct depending on how $\mathrm{n}$ and $E^{\circ}$ are defined, but the apparent contradiction has led some textbooks to incorrectly describe the $\mathrm{y}$-axis as proportional to $-\Delta G^{\circ} / \mathrm{F}, 6,12$ which is unambiguously incorrect. We aim to highlight these contradictory conventions and suggest ways to avoid confusion over the correct quantitative relationship between free energy and reduction potentials.

A more substantial error concerns the interpretation of Frost diagrams. Many undergraduate textbooks instruct students to regard the lowest point on a Frost diagram as the most stable oxidation state of an element, but this statement is incorrect, as becomes apparent if the reference potential of 
each redox couple is changed. As illustrated in Figure 2, re-referencing the reduction potentials from the standard hydrogen electrode (SHE) to the $\mathrm{O}_{2} / \mathrm{H}_{2} \mathrm{O}$ redox couple alters which oxidation state is lowest on the diagram. Since reference potentials are arbitrary points for comparing different half reactions, this observation invalidates the notion that the lowest point represents the most stable oxidation state, at least not in an absolute sense.

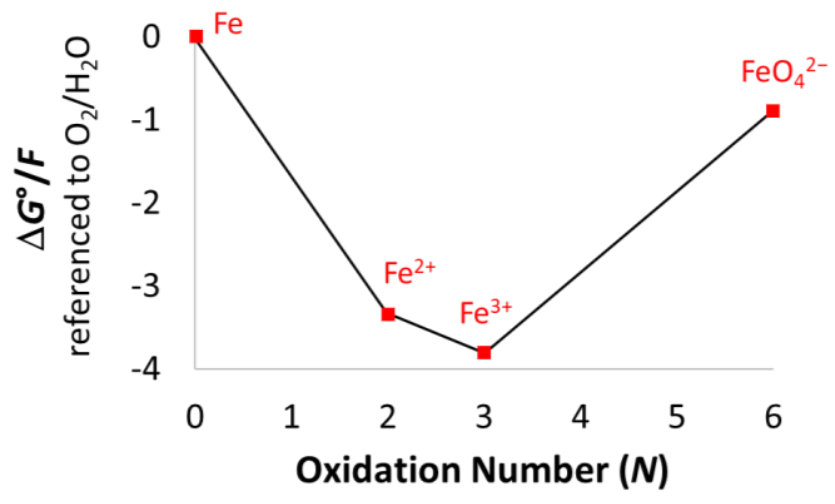

Figure 2. Frost diagram for iron at $\mathrm{pH}=0$ with reduction potentials referenced to $\mathrm{O}_{2} / \mathrm{H}_{2} \mathrm{O}$ couple.

Instead, the lowest point in a typical Frost diagram represents the favored oxidation state of an element under conditions that apply a potential equal to that of the standard hydrogen electrode. We aim to clarify this misconception introduced by many textbooks and highlight how the correct interpretation adds greater utility to these diagrams since changing the reference potential provides a way to illustrate how the favored oxidation state changes based on the specific conditions present in solution. To aid this effort, we introduce interactive Frost diagrams in which the applied potential $\left(E_{\text {app }}\right)$ and $\mathrm{pH}$ can be varied arbitrarily to illustrate how the favored oxidation state of an element changes.

\section{CORRECTING MISCONCEPTIONS}

Y-Axis Free Energy Label.

In the 1951 paper introducing Frost diagrams ${ }^{1}$, Arthur Frost plotted free energy in electronvolts $(\mathrm{eV})$ on the $\mathrm{y}$-axis versus the oxidation number of an element on the $\mathrm{x}$-axis. Organizing the data in this manner positions the thermodynamically favored oxidation state of an element (relative to $\mathrm{H}^{+} / \mathrm{H}_{2}$ ) as the lowest point on the diagram, thus adhering to the usual convention of depicting lower energy species beneath higher energy species. For instructive purposes, it has become common to label the y-axis in terms of electrode potentials and the number of electrons separating each oxidation state from the standard $N=0$ state of an element. This labeling is proportional to free energy based on Equation 1, where $\mathrm{n}$ is the number of electrons involved in a redox process, $E^{\circ}$ is the standard electrode potential in volts, and $\Delta G^{\circ} / F$ is the standard free energy change in $\mathrm{eV}$ (note: $\Delta G / F$ and $E$ apply when $\mathrm{pH} \neq 0$ or 14 ). 


$$
-n E^{\circ}=\Delta G^{\circ} / F
$$

Thus, it would seem that the y-axis of Frost diagrams should be labeled $-\mathrm{n} E^{\circ}$ to correspond to $\Delta G^{\circ} / F$,

80 and this convention is common (Figure 3A). ${ }^{15-18}$ However, this is only correct if $E^{\circ}$ is defined as the actual potential for the conversion of the $N=0$ oxidation state to the other oxidation states in question, meaning that $E^{\circ}$ could be either a reduction potential $\left(E^{\circ}\right.$ red $)$ or an oxidation potential $\left(E_{\text {ox }}^{\circ}\right.$, where $E_{\text {ox }}^{\circ}=-E_{\text {red }}^{\circ}$. This can create confusion since the IUPAC defines $E^{\circ}$ to refer specifically to standard reduction potentials (i.e., $E^{\circ}=E^{\circ}$ red).

A common alternative convention labels the $\mathrm{y}$-axis of Frost diagrams as $\mathrm{n} E^{\circ},{ }^{4-}$ 14,19 in which $\mathrm{n}$ equals the oxidation state along the $\mathrm{x}$-axis and $E^{\circ}$ is exclusively a standard reduction potential. For oxidation states $<0$, this convention reproduces the relationship of Eq 1 since the negative sign is introduced by the $N<0$ value of the oxidation state. For oxidation states $>0$, the use of $E^{\circ}$ $=E^{\circ}$ red introduces the negative sign since the free energy change of an oxidative process should be calculated using the standard oxidation potential as $E^{\circ}$ in $\mathrm{Eq} 1$ and $E_{\text {red }}^{\circ}=$ $-E_{\text {ox. }}^{\circ}$ Thus, this convention correctly sets the proportionality of the y-axis to free energy but

\section{A Common valid $y$-axis Labels:}

(1) $\Delta \boldsymbol{G}^{\circ} / \boldsymbol{F}=-\mathrm{n} \boldsymbol{E}^{\circ}$ where $\mathrm{n}=$ number of electrons $E^{\circ}=$ standard reduction potential for

$$
[\mathrm{N}=0] \rightarrow[\mathrm{N}<0]
$$

or

standard oxidation potential for $[\mathrm{N}=0] \rightarrow[\mathrm{N}>0]$

Better: $\Delta G^{\circ} / F=-n E^{\circ}{ }_{r x n}$ where " $E^{\circ}{ }^{\prime}$ " replaces " $E^{\circ "}$ for potentials

(2) $\Delta \boldsymbol{G}^{\circ} / \boldsymbol{F}=\mathrm{n} \boldsymbol{E}^{\circ}$ where $\mathrm{n}=$ oxidation state

$$
\begin{aligned}
& E^{\circ}=\text { standard reduction potential for } \\
& {[\mathrm{N}=0] \rightarrow[\mathrm{N}<0] \text { and }[\mathrm{N}>0] \rightarrow[\mathrm{N}=0]}
\end{aligned}
$$

Better: $\Delta G^{\circ} / F=N E^{\circ}$ where " $N$ " replaces " $\mathrm{n}$ " for oxidation state

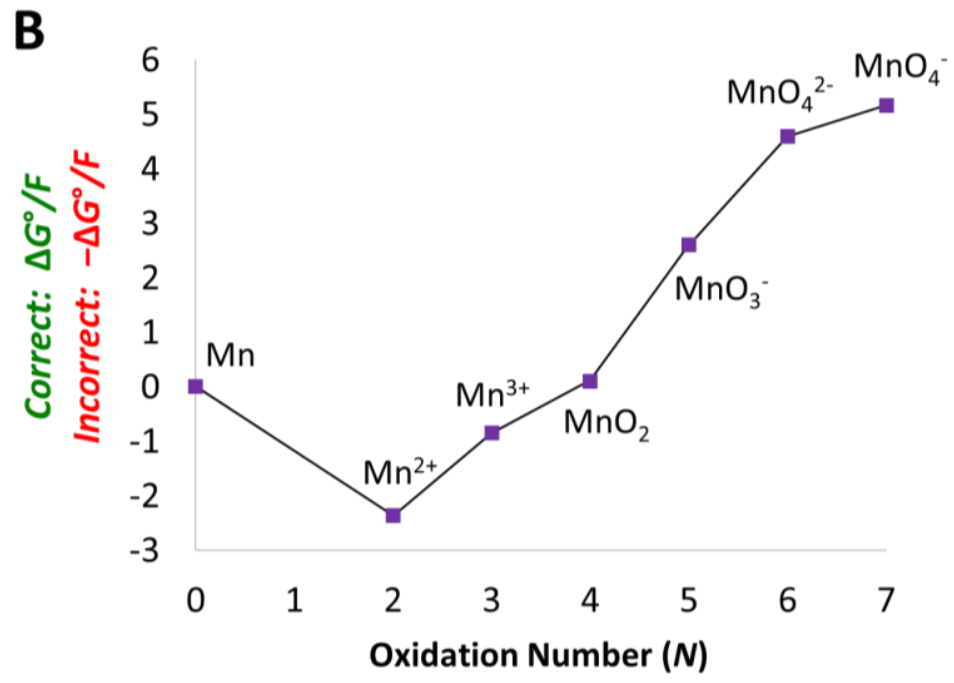

Figure 3. (A) Common conventions for labeling the y-axis of Frost diagrams and suggested clarifications. (B) Frost diagram for manganese with the correct energy label for the $\mathrm{y}$-axis (green) and a common incorrect (red) y-axis label.

can produce confusion by representing $\Delta G^{\circ} / F$ as equal to $\mathrm{n} E^{\circ}$. Indeed, this confusion has led more than one textbook to incorrectly label the y-axis of Frost diagrams as representing $-\Delta G^{\circ} / F$ (Figure $3 \mathrm{~B}$ ), which cannot be justified regardless of how terms are defined. 
One common way of alleviating the above confusion is to use $N$ to represent the oxidation state in the y-axis label $N E^{\circ}$ to ensure that the signed oxidation state term is not confused with the unsigned term $\mathrm{n}$ that represents the number of electrons in Eq 1.20 This solution has the advantage of simplicity since $N$ in the y-axis label $N E^{\circ}$ is also used to label the x-axis, and $E^{\circ}$ represents only standard reduction potentials $E_{\text {red. }}^{\circ}$ However, we advocate for a different clarified y-axis label of $-\mathrm{n} E^{\circ}{ }_{\text {rxn }}$ where $\mathrm{n}$ is the number of electrons as in Eq 1, and $E_{\text {rxn }}^{\circ}$ is the reduction potential $\left(E_{\text {red }}^{\circ}\right)$ for movement to $N<0$ oxidation states or the oxidation potential $\left(E^{\circ}{ }_{\text {ox }}\right)$ for movement to $N>0$ oxidation states. Though this solution is a little more complex, it serves to better reinforce the relationship between potentials and free energy described in Eq 1. Additionally, the use of $E_{\text {rxn }}^{\circ}$ highlights that changes of an element's oxidation state must be accompanied by the opposite change of some other redox couple to provide a net redox reaction. In other words, $E_{\text {rxn }}^{\circ}$ is a cell potential for a full redox reaction, as required to meaningfully compare energy changes. As discussed in the next section, this latter point is often overlooked in explanations of Frost diagrams since the $\mathrm{H}^{+} / \mathrm{H}_{2}$ couple is hidden by its role as the standard $\mathrm{O} \mathrm{V}$ reference potential.

\section{Conditional Stability of Oxidation States.}

Many textbooks describe the lowest point on a Frost diagram as the most stable oxidation state of the element in water at $\mathrm{pH}=0$ (or for whichever $\mathrm{pH}$ the diagram was constructed), but this interpretation is incorrect, and a more nuanced explanation is needed: the lowest point corresponds to the thermodynamically favored species relative to the $\mathrm{H}^{+} / \mathrm{H}_{2}$ redox couple that is used as the $E^{\circ}=0 \mathrm{~V}$ reference for tabulating standard electrode potentials. As explained by Frost, “a positive slope [connecting two oxidation states] means a tendency for the couple to oxidize $\mathrm{H}_{2}$ to $\mathrm{H}^{+}$while a negative slope shows a tendency towards reduction [of $\mathrm{H}^{+}$to $\mathrm{H}_{2}$ ]." In other words, the free energy change measured on the y-axis of a Frost

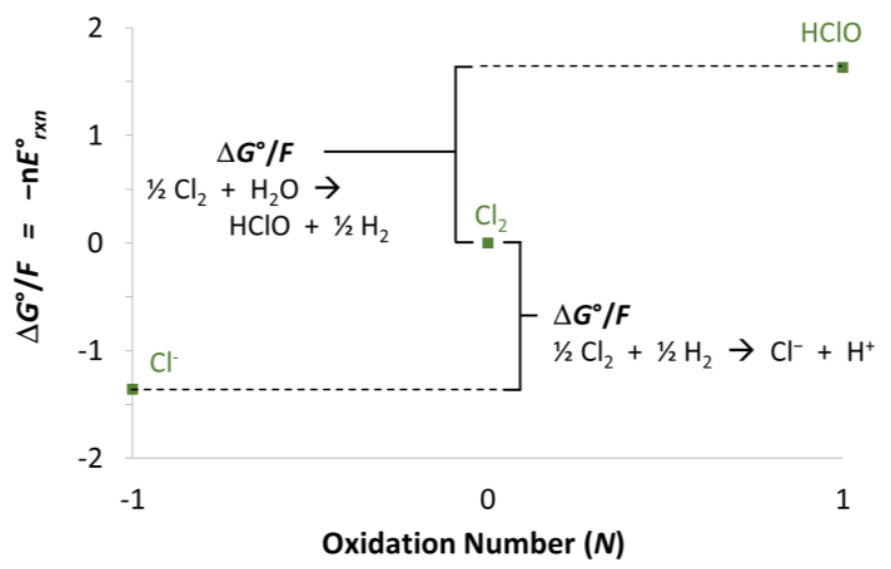

Figure 4. Partial Frost diagram for chlorine illustrating the full redox reactions connecting the oxidation states. which the oxidation or reduction of the $N=0$ state of an element is paired with the reduction of $\mathrm{H}^{+}$to $\mathrm{H}_{2}$ or oxidation of $\mathrm{H}_{2}$ to $\mathrm{H}^{+}$, respectively (Figure 4). Textbooks rarely discuss this feature of the diagrams, 
and many inaccurately describe the lowest point on a Frost diagram as the most stable oxidation state of an element. However, as revealed in comparing Figures 1 and 2, changing the reference potential gives rise to interchangeable lowest points, which reflect that the favored oxidation state is dependent on the specific redox conditions in solution (i.e., the potential applied by other redox-active species or by an electrode). Thus, the conclusion that the lowest point is the most stable is only conditionally true, and in fact, is meaningless in an absolute sense since half reactions must be paired with other half reactions to determine the overall thermodynamic driving force.

It is important to emphasize the significance of the reference redox couple used to construct Frost diagrams in order to reinforce that the free energy change of a redox process depends on a net reaction, not just the potentials of individual half reactions. Failing to emphasize this point causes confusion that extends beyond students in the classroom. We have seen this misconception in publications $^{21}$ from researchers in the field of electrochemistry, suggesting that textbooks are introducing a persistent misunderstanding. Labeling the $\mathrm{y}$-axis $-\mathrm{n} E_{\mathrm{rxn}}^{\circ}$ helps resolve this misconception by emphasizing that the change in free energy involves a net redox process as noted in Frost's original paper. The term $E_{\text {rxn }}^{\circ}$ is also useful since it is nonspecific, allowing consideration of how Frost diagrams change under different applied potentials. The interactive diagrams introduced in the next section allow users to change the applied potential to immediately observe changes, which highlights how the favored oxidation state of an element is context dependent.

\section{INTERACTIVE FROST DIAGRAMS}

Textbooks often present Frost diagrams for different $\mathrm{pH}$ conditions to illustrate the effects of $\mathrm{pH}$ on redox processes (Figure 5A,B), but rarely are the effects of the applied potential noted in these diagrams. We present Frost diagrams for four elements (N, Cl, Fe, and Mn) in the form of Microsoft Excel spreadsheets in which the reduction potentials connecting each oxidation state are recalculated upon changing the applied potential or $\mathrm{pH}$, thus providing an interactive visualization of how the 
thermodynamics of redox processes are affected (Figure 5C). Since the favored protonation state can example, two oxidation states are equally favorable (i.e., the two points are at equal heights on the yaxis) at an applied potential equal to the reduction potential separating them, as illustrated for the $N=$ 0 and $N=-3$ states in Figure 5C.

conditional, while some properties, such as tendencies towards disproportionation and comproportionation are independent of potential (though can be dependent on $\mathrm{pH}$ ). The interactive diagrams also illustrate the important general relationship between reduction potentials, applied potentials, and thermodynamic equilibria. For
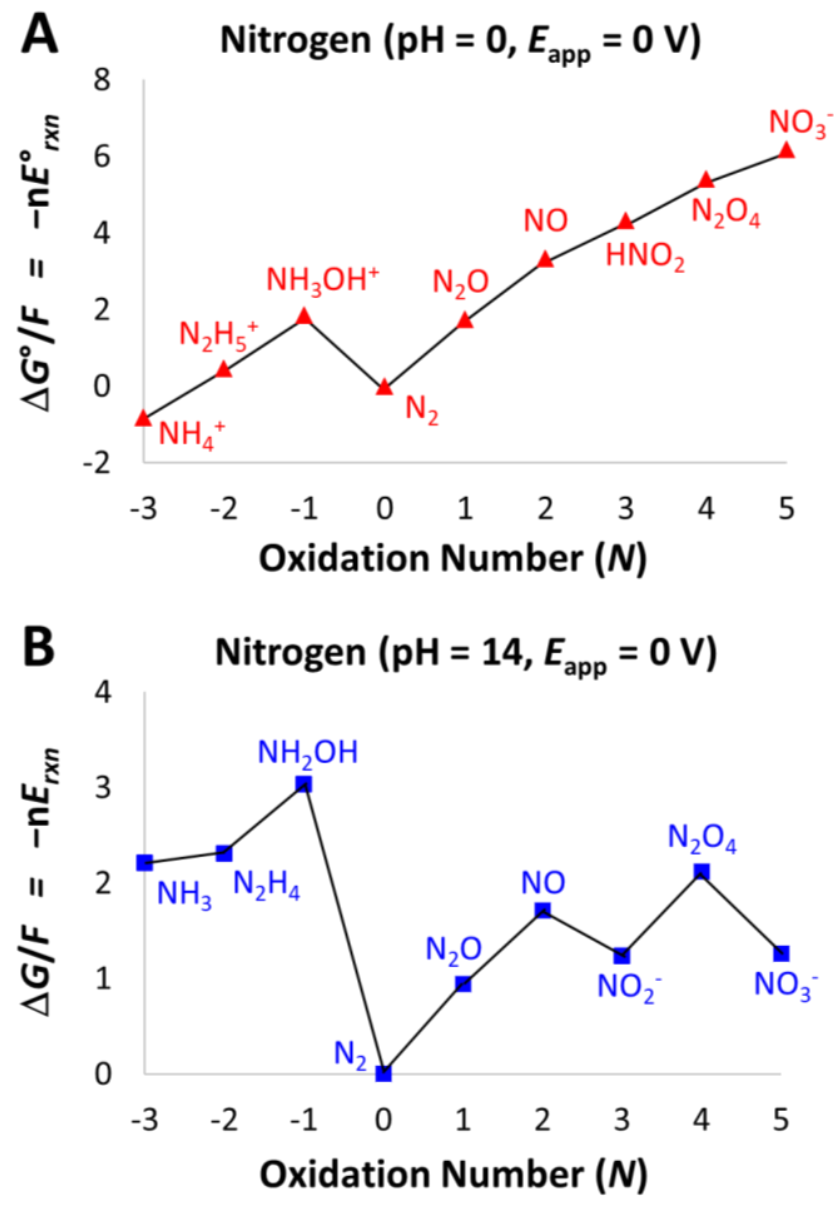
(B) Frost diagram for nitrogen modified to represent $\mathrm{pH}=14$ conditions. (C) A customized Frost diagram for nitrogen representing conditions $\left(\mathrm{pH}=9.27, E_{\text {app }}\right.$ $=-0.4575 \mathrm{~V}$ vs. SHE) in which $\mathrm{NH}_{4}{ }^{+}, \mathrm{NH}_{3}$, and $\mathrm{N}_{2}$ are equal in energy. 


\section{Box 1: Using the Interactive Frost Diagrams}

We provide interactive Frost Diagrams as a .xls file containing a sheet for each of four selected elements (nitrogen, chlorine, iron, manganese). There are tables on each sheet to represent acidic species (in red) and basic species (in blue). Redox couples and standard potentials from Latimer diagrams are tabulated to the left, while the righthand tables show individual species for each oxidation state along with $E_{\mathrm{rxn}}$ potentials and $-\mathrm{n} E_{\mathrm{rxn}}$ energies that are recalculated in response to changes in $\mathrm{pH}$ and applied potential $\left(E_{\mathrm{app}}\right)$. Cells for $\mathrm{pH}$ and $E_{\mathrm{app}}$ are highlighted in yellow. Users need only adjust these values since changes are propagated through the tables. The example shown here was used to calculate the Frost Diagram in Figure $5 \mathrm{C}$ in which $\mathrm{N}_{2}, \mathrm{NH}_{3}$, and $\mathrm{NH}_{4}{ }^{+}$are equal in energy.

\begin{tabular}{|c|c|c|c|c|c|c|c|c|}
\hline & & & & $\mathrm{pH}=$ & 9.27 & & $E$ app $=$ & -0.4575 \\
\hline \multicolumn{9}{|l|}{ Acidic } \\
\hline Redox Couples & \# e- & $\# \mathrm{H+}$ & $E^{\circ}$ red & Species & Ox.\#(N) & E rxn (1 step) & $E$ rxn (from $\mathrm{N}=0$ ) & $-n E r x n$ \\
\hline NO3(1-)/N2O4 & 1 & 2 & 0.798 & NO3 (1-) & 5 & -0.162 & -1.046 & 5.230 \\
\hline N2O4/HNO2 & 1 & 1 & 1.07 & N2O4 & 4 & -0.981 & -1.267 & 5.068 \\
\hline $\mathrm{HNO} 2 / \mathrm{NO}$ & 1 & 1 & 0.996 & HNO2 & 3 & -0.907 & -1.363 & 4.088 \\
\hline $\mathrm{NO} / \mathrm{N} 2 \mathrm{O}$ & 1 & 1 & 1.59 & NO & 2 & -1.501 & -1.591 & 3.181 \\
\hline $\mathrm{N} 2 \mathrm{O} / \mathrm{N} 2$ & 1 & 1 & 1.77 & $\mathrm{N2O}$ & 1 & -1.681 & -1.681 & 1.681 \\
\hline $\mathrm{N} 2 / \mathrm{NH} 3 \mathrm{OH}(1+)$ & 1 & 2 & -1.87 & $\mathrm{~N} 2$ & 0 & 0 & 0 & 0 \\
\hline $\mathrm{NH} 3 \mathrm{OH}(1+) / \mathrm{N} 2 \mathrm{H} 5(1+)$ & 1 & 0.5 & 1.41 & NH3OH (1+) & -1 & -2.506 & -2.506 & 2.506 \\
\hline \multirow[t]{2}{*}{$\mathrm{N} 2 \mathrm{H} 5(1+) / \mathrm{NH} 4(1+)$} & 1 & 1.5 & 1.275 & $\mathrm{~N} 2 \mathrm{H} 5(1+)$ & -2 & 1.594 & -0.456 & 0.912 \\
\hline & & & & $\mathrm{NH} 4(1+)$ & -3 & 0.912 & 0.000 & 0.000 \\
\hline \multicolumn{9}{|l|}{ Basic } \\
\hline Redox Couples & \# e- & \# H2O/OH- & $E^{\circ}$ red & Species & Ox. \# (N) & Erxn (1 step) & $E$ rxn (from $\mathrm{N}=0$ ) & $-n E r x n$ \\
\hline NO2(1-)/ NO & 1 & 2 & -0.46 & NO2 (1-) & 3 & -0.556 & -1.246 & 3.737 \\
\hline $\mathrm{NO} / \mathrm{N} 2 \mathrm{O}$ & 1 & 1 & 0.764 & NO & 2 & -1.501 & -1.591 & 3.181 \\
\hline $\mathrm{N} 2 \mathrm{O} / \mathrm{N} 2$ & 1 & 1 & 0.944 & $\mathrm{N2O}$ & 1 & -1.681 & -1.681 & 1.681 \\
\hline $\mathrm{N} 2 / \mathrm{NH} 2 \mathrm{OH}$ & 1 & 1 & -3.04 & N2 & 0 & 0 & 0 & 0 \\
\hline $\mathrm{NH} 2 \mathrm{OH} / \mathrm{N} 2 \mathrm{H} 4$ & 1 & 1 & 0.73 & $\mathrm{NH} 2 \mathrm{OH}$ & -1 & -2.303 & -2.303 & 2.303 \\
\hline \multirow[t]{2}{*}{$\mathrm{N} 2 \mathrm{H} 4 / \mathrm{NH} 3$} & 1 & 1 & 0.1 & $\mathrm{~N} 2 \mathrm{H} 4$ & -2 & 1.467 & -0.418 & 0.837 \\
\hline & & & & NH3 & -3 & 0.837 & 0.000 & 0.000 \\
\hline
\end{tabular}

The interactive diagrams also allow the influence of $\mathrm{pH}$ on the thermodynamics of redox processes to be observed. Overlaying the diagrams derived from acidic and basic species allows users to to compare the free energies of different protonation states for weak acids at a desired $\mathrm{pH}$. The overlaid diagrams show that protonated weak acids are favored at lower $\mathrm{pH}$, while the conjugate bases become favorable as $\mathrm{pH}$ increases. If the $\mathrm{pH}$ is set to the $\mathrm{pK}_{\mathrm{a}}$ of an acid in the diagram, the points for the acid and its conjugate base overlap (Figure 5C), illustrating that the acid and base are equal in energy at a $\mathrm{pH}$ matching the $\mathrm{pK}_{\mathrm{a}}$ of the acid, similar to the observation that two oxidation states are equal in energy at a potential matching the reduction potential between the two oxidation states. Observing that $\mathrm{pH}$ and $E_{\text {app }}$ both similarly affect the Frost diagrams illustrates that there is a similar thermodynamic relationship between reduction potentials and applied potentials as there is between $\mathrm{pK}_{\mathrm{a}}$ and $\mathrm{pH}$. 
Connection to Pourbaix Diagrams.

Pedagogically, the interactive Frost diagrams are particularly useful when combined with Pourbaix diagrams (Figure 6) since students can cross-reference potentials and $\mathrm{pH}$ on a Pourbaix diagram with the Frost diagrams generated for those conditions. The lowest species depicted in the Frost diagram will correspond to the favored species indicated in the Pourbaix diagram for a given potential and $\mathrm{pH}$. Furthermore, these Frost diagrams add nuance to the Pourbaix

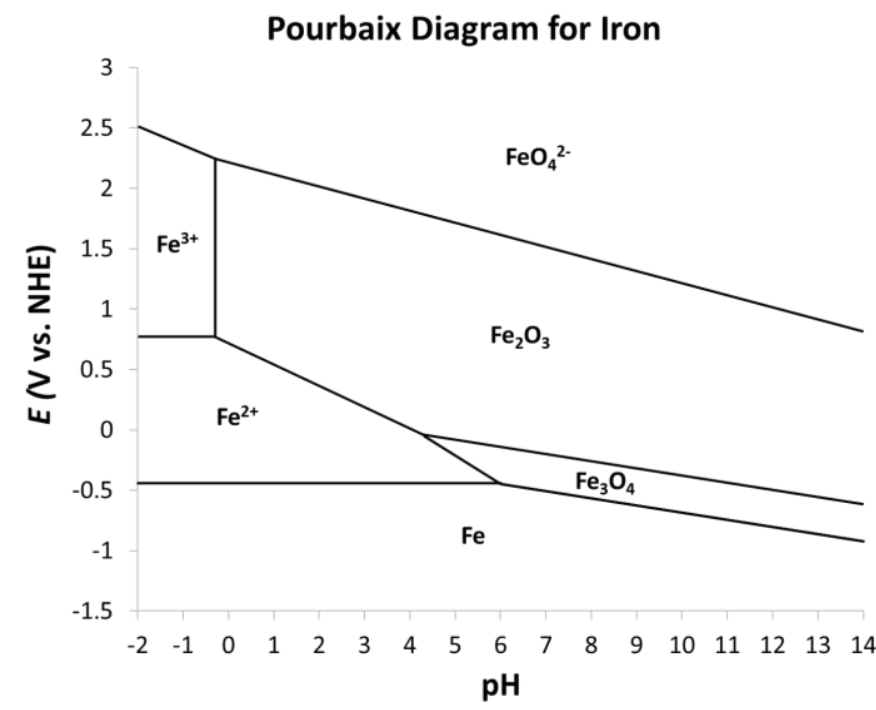

Figure 6. Pourbaix diagram for iron. ${ }^{22-24}$ diagrams by illustrating how the equilibrium distribution between multiple species changes under different conditions, rather than just indicating the single most favorable species. For example, the Frost diagrams show that any points on a line separating two regions in the Pourbaix diagram represent conditions in which the two oxidation states and/or protonation states are equal in energy. Likewise, points in the Pourbaix diagram in which three lines intersect will show as having three equal energy species in the Frost diagram at that $\mathrm{pH}$ and potential.

\section{CONCLUSIONS}

We have identified two common errors regarding the labeling and interpretation of Frost diagrams that have been perpetuated by many textbooks and other resources. To aid in correcting these errors, we have developed a series of interactive Frost diagrams that illustrate the dependence of the relative free energies of oxidation states of an element on $\mathrm{pH}$ and the redox conditions in solution (i.e., the potential applied by an electrode or the environment). The y-axes of the interactive diagrams are labeled correctly to be proportional to the free energy of each oxidation state of the element under different applied potentials. Instructors can use these diagrams as pedagogical tools to complement Pourbaix diagrams while also reinforcing how reduction potentials relate to the concept of chemical equilibrium. 


\section{ASSOCIATED CONTENT}

Supporting Information available:

Notes for Instructors, Exercise Questions, and Answer Key (PDF, DOCX)

Interactive Frost Diagrams (XLS)

\section{AUTHOR INFORMATION}

Corresponding Author

*E-mail: m11353@chem.rutgers.edu

\section{ACKNOWLEDGMENTS}

The authors acknowledge Rutgers, The State University of New Jersey, for generous financial support.

\section{REFERENCES}

1. Frost, A. A.; Oxidation Potential-Free Energy Diagrams. J. Am. Chem. Soc.. 1951, 73, 2680-2682.

2. Latimer, W. M. The oxidation states of the elements and their potentials in aqueous solutions, 1 ed.; Prentice-Hall: New York, 1938.

3. Delahay, P., Pourbaix, M., and Van Rysselberghe, P.; Potential-pH diagrams. J. Chem. Educ. $195027,683-688$.

4. Phillips, C. S. G. and Williams, R. J. P.; Inorganic chemistry. 1 ed.; Oxford University Press: New York, 1965.

5. Moeller, T.; Inorganic Chemistry, a Modern Introduction. Wiley: New York, 1982.

6. Housecraft, C. E. and Sharpe, A.; Inorganic Chemistry. 2 ed.; Prentice-Hall: New York, 2005.

7. Miessler, G. L., Fischer, P.J., and Tarr, D. A.; Inorganic Chemistry. 5 ed.; Pearson: New York, 2014.

8. Cox, P. A. and Cox, T.; Inorganic Chemistry. Bios Scientific Publishers: England, 2004.

9. Mackay, K. M. and Mackay, R. A.; Introduction to Modern Inorganic Chemistry. 2 ed.; Intertext books; 1974.

10. Mackay, K. M. and Mackay, R. A.; Introduction to Modern Inorganic Chemistry. 3 ed.; International Textbook; 1981.

11. Mackay, K. M. and Mackay, R. A.; Introduction to Modern Inorganic Chemistry. 4 ed.; Blackie; 1989.

255 12. Shriver, D. F., Weller, M., Overton, T. Rourke, J., and Armstrong, F.; Inorganic Chemistry. 6 ed.; W. H. Freeman: New York, 2014. 
13. Martinez de llarduya, J. M. and Villafane, F.; A Warning to Frost Diagram Users. J. Chem. Educ. 1994, 71, 480-482.

14. Chemistry LibreTexts: 4.4 Latimer and Frost Diagrams. https://chem.libretexts.org/@go/page/183313 (accessed Mar 2021).

15. Emeléus, H. J. and Sharpe, A. G.; Modern Aspects of Inorganic Chemistry. 4 ed.; Wiley: New York, 1973.

16. Mackay, K. M. and Mackay, R. A.; Introduction to Modern Inorganic Chemistry. 1 ed.; Intertext books; 1969.

17. Barrett, J. and Malati, M. A.; Fundamentals of Inorganic Chemistry: An Introductory Text for Degree Course Studies. 1 ed.; Horwood Publishing Ltd.: England, 1998.

18. Rayner-Canham, G. and Overton, T.; Descriptive Inorganic Chemistry. 5 ed.; W. H. Freeman: New York, 2010.

19. Interestingly, we have found one instance of a text that had labeled the $y$-axis $-n E^{\circ}$ in its first edition (reference 16) but switched to $\mathrm{n} E^{\circ}$ in later editions (reference 9).

20. Chemistry LibreTexts: 8.1.4.2. Frost Diagrams show how stable element's redox states are relative to the free element. https://chem.libretexts.org/@go/page/199665 (accessed Apr 2021).

21. González Pérez, O. and Bisang, J. M.; Electrochemical Removal of Nitrite Using an Activated Copper Rotating Cylinder Electrode. J. Electrochem. Soc. 2017, 164, E300-306.

22. The Pourbaix diagram for iron presented in Figure 6 and the interactive Frost diagram for iron provided as supporting information were constructed using reduction potentials tabulated in reference 23. These data produce a $\mathrm{pH}$ value $(-0.28)$ separating the $\mathrm{Fe}^{3+}$ and $\mathrm{Fe}_{2} \mathrm{O}_{3}$ states that is much lower than commonly presented in Pourbaix diagrams for iron. This difference is likely because the data from reference 23 uses $\mathrm{Fe}^{3+}$ to refer specifically to $\left[\mathrm{Fe}\left(\mathrm{OH}_{2}\right)_{6}\right]^{3+}$, which exists only at very low $\mathrm{pH}$, while other soluble $\mathrm{Fe}(\mathrm{III})$ species exist at higher $\mathrm{pH}$ before the precipitation of $\mathrm{Fe}_{2} \mathrm{O}_{3}$. In general, $\mathrm{Fe}(\mathrm{III})$ exhibits complex speciation in aqueous solution and as solid oxides, resulting in many acceptable variations of the Pourbaix diagram for iron. Reference 24 presents a Pourbaix diagram for iron that is consistent with the one we constructed.

23. Bratsch, Steven G.; Standard Electrode Potentials and Temperature Coefficients in Water. J. Phys. Chem. Ref. Data, 1989, 18, $1-21$.

24. Perry, S. C., Gateman, S. M., Stephens, L. I., Lacasse, R., Schulz, R., and Mauzeroll, J.; Pourbaix Diagrams as a Simple Route to First Principles Corrosion Simulation. J. Electrochem. Soc. 2019, 166, C3186 - C3192. 